\title{
Psycho-emotional disorders in postpartum women after stillbirth
}

\section{Gustavo Romero-Gutiérrez*, Nadia Berenice Bracamontes-Bayardo, Armando Ruiz-Treviño}

Dept Obstetrics and Gynecology UMAE Hospital de Gineco-Pediatría № 48, IMSS, León, Guanajuato, México

\section{Email address:}

gustavoromerog@hotmail.com (G. Romero-Gutiérrez,), n3b84@hotmail.com (N. B. Bracamontes-Bayardo), armando.ruiz.t@gmail.com (A. Ruiz-Treviño)

\section{To cite this article:}

Gustavo Romero-Gutiérrez, Nadia Berenice Bracamontes-Bayardo, Armando Ruiz-Treviño. Psycho-Emotional Disorders in Postpartum Women after Stillbirth. Journal of Gynecology and Obstetrics. Vol. 2, No. 3, 2014, pp. 32-36. doi: 10.11648/j.jgo.20140203.11

\begin{abstract}
Objective: To identify psycho-emotional disorders diagnosed in women after stillbirth. Design: Cross-sectional study a face-to-face questionnaire. Setting: Postpartum area at a tertiary care referral hospital in León, Guanajuato, México. Participants: 210 women at immediate postpartum, divided into 105 women with stillbirth and 105 with healthy live newborn (controls). Data collection: Women answered face-to-face the questionnaire Minnesota Multiphase Personality Inventory-2. Findings: In $34.2 \%$ of women who had stillbirth, were diagnosed psycho-emotional disorders, compared with controls who were found in $19.0 \%, \mathrm{p}=0.019$. In the logistic regression analysis the only significant risk factor associated to psycho-emotional disorder was the presence of fetal death, $\mathrm{p}=0.002$. The psycho-emotional disorders found in women with stillbirth were: depression $(p=0.026)$, hysteria $(p=0.012)$, psychotic deviation $(p=0.007)$, paranoia $(p=0.013)$, psychasthenia $(p=0.034)$, schizophrenia $(p=0.017)$, low self-esteem $(p=0.044)$ and anxiety $(p=0.049)$. Key conclusions: There is higher frequency of psycho-emotional disorders in women who suffer fetal death. Implications for practice: It is recommended to provide early care and psychological support to women who deliver a stillbirth.
\end{abstract}

Keywords: Fetal Death, Stillbirth, Minnesota Multiphase Personality Inventory-2 (Mmpi-2), Psycho-Emotional Disorders

\section{Introduction}

Fetal death is a serious public health problem; it is a devastating obstetric outcome that creates a major impact on the health team involved as well as the family environment [1]. Worldwide, 2.64 million of fetal deaths occur each year. In our country it is estimated a rate of 4.5 deaths per 1,000 births [2], moreover $98 \%$ of fetal deaths occur in developing countries and a total of 1.19 million occurs intrapartum [3]. According to the American Colleague of Obstetrics and Gynecology (ACOG), the fetal death or stillbirth is defined as the cessation of fetal life from 20 weeks of gestation or fetal weight of 350 grams or more [4].

In most cases the etiology of fetal death is unknown, the risk factors associated with stillbirth are maternal age over 35 or under 20 years; obesity; hypertensive disease of pregnancy; diabetes mellitus; drug addiction; primiparity; poor access to medical care; low level of education; history of fetal loss and intrauterine growth restriction [5-7]. Some investigations have reported that 20 to $30 \%$ of women with fetal death present a long-term psychiatric disorder [8].
Similarly, it has been reported that the loss of an infant from stillbirth, abortion or neonatal death is recognized as a traumatic event. Stillbirth parents inevitably triggers a process of mourning and grief that may last a year or more. Couples and their families often experience feelings of anxiety, failure, frustration, guilt and apprehension, making necessary psychological support [9].

Some studies have established that women who suffered a stillbirth may be vulnerable to experiencing symptoms of depression, anxiety and posttraumatic stress in the next pregnancy and subsequent postpartum, particularly when pregnancy occurs early after the loss $[10,11]$. In recent years there have been various researches regarding the psychological impact in patients who suffer fetal death. Despite, in our country, as well as in other developing countries, there are no studies that evaluate the psycho-emotional disorders that present women experiencing this devastating obstetric complication. Our aim was to identify the frequency of psycho-emotional disorders in postpartum women who suffered fetal death. 


\section{Methods}

\subsection{Design and Settings}

A cross-sectional study was conducted at the Hospital of Obstetrics and Gynecology at the Mexican Institute of Social Security located in León, Guanajuato, México, from February 2011 to July 2013. During this period, the patient recruitment was through a non-probability sampling of consecutive cases. The study protocol was approved by the ethics and research committee of the local institutional review board; and women who agreed to participate in the study completed a written informed consent form. The recruited women answered face-to-face questions from the Minnesota Multiphasic Personality Inventory-2 (MMPI-2), [12] applied at immediate postpartum.

\subsection{Study Participants}

Two hundred and ten women were divided into two groups: One hundred and five women who had a stillbirth and one hundred and five women who had a healthy live newborn (controls). The women were recruited according to the following:

Inclusion criteria: All patients with fetal death according to criteria of the ACOG [4], regardless of age, number of gestations, previous abortions or previous fetal deaths and medical complications that occur in our hospital.

Exclusion criteria: Patients with pregnancies less than 20 weeks or newborns weighting less than 350 gr. and women who did not obtain informed consent.

Elimination Criteria: Patients whose answers showed inconsistencies and met the criteria for determining disability according the psychological scale MMPI-2.

\subsection{Instruments}

The psychological assessment consisted in clinical interviews and administration of MMPI-2. The test is a recent version of the original MMPI that was designed to help identify personal, social, and behavioral problems in psychiatric patients. This MMPI-2 edition can be used in healthy individuals, and this questionnaire has been validated in a population of pregnant women [13].

It consists of 42 scales, a total of 567 reagents true or false response ordered so as to allow the qualification of the basic scales (clinical and validity) with the first 370 reagents. Content profile include scales of anxiety, fear, anger, low self-esteem and scale supplementary profile the scale of post-traumatic stress; a total of 14 scales. It calculates an estimated time of 60-90 minutes with each patient to their application. Conventionally, it was considered the presence of 3 or more alterations in one patient as having psycho-emotional disorder.

Because of possible participation of regional characteristics, we evaluated some socio-demographic variables of our population, as well as clinical factors; the factors registered were: maternal age, which was defined as completed years at the time of delivery; marital status, which was registered as 0 if the women was currently married and as 1 if the women had never married or was divorced, separated, or widowed (single women); education level, which was registered as the number of completed years of school; women occupation, which was categorized as 0 if the woman had no formal occupation and as 1 if she had employment outside the home (working women); parity, which was defined as the number of previous births; weeks of gestation; previous pregnancies, including abortions and stillbirths; antenatal care, which was categorized as 0 when the woman received no antenatal care at the hospital and 1, when she attended at least one appointment in the antenatal care department; the number of antenatal visits for each woman, coded as the current number of prenatal appointments during the pregnancy; acceptance of a contraceptive method after the present pregnancy; and the following variables were registered conventionally as yes $=$ 1 or not $=0$, according to responses of women about: enough and accurate medical advice during antenatal care; family support during pregnancy; drug addictions; interfamilial violence and obstetric complications.

\subsection{Data Analysis}

Data were analyzed using descriptive statistical procedures which included arithmetic mean, standard error (SE) and percentages. The groups were compared using the Chi-square analysis or Fisher Exact' test for categorical variables; and Student t' test or Mann-Whitney U test for continuous variables as appropriate depending on their normal distribution, the Alpha value was set at 0.05 . Logistic regression analysis was performed to find predictors of psycho-emotional disorders, considering as regressors the variables socio-demographic and clinical. Statistical analysis was performed with the Number Cruncher Statistical System computer program [14].

\section{Results}

The maternal mean age was $27.9 \pm 0.6$ years in women with fetal death, with a range between 15 and 44 years, while age of the women with healthy newborn was $24.9 \pm$ $0.4, p=0.001$. The $74.3 \%$ of the cases occurred in patients between 20 and 34 years. The frequency of mothers over 35 years was higher in the case group (18 vs. $3, \mathrm{p}=0.001)$. More women with stillbirths were married ( 99 vs. $89, \mathrm{p}=$ $0.043)$. Women with stillbirths had less education level (9.8 vs. 10.6 years, $p=0.017$, (table 1 ). In the analysis of clinical variables, the gestational age in women with stillbirths ranged from 20-41 weeks, with an average of $30.1 \pm 0.6$ weeks. The $40 \%$ of fetal death occurred early ( 20 to 27.6 weeks gestation) and $60 \%$ late (>28 weeks). 
Table 1. Demographic variable analysis of the two groups. [Mean $\pm S E ; n$ $(\%)]$

\begin{tabular}{llll}
\hline Variable & $\begin{array}{l}\text { Cases } \\
(\mathbf{n}=\mathbf{1 0 5})\end{array}$ & $\begin{array}{l}\text { Controls } \\
(\mathbf{n}=\mathbf{1 0 5})\end{array}$ & $\mathbf{p}$ \\
\hline Maternal age (years) & $27.9 \pm 0.6$ & $24.9 \pm 0.4$ & 0.001 \\
$<$ 20 years & $9(8.5)$ & $12(11.4)$ & 0.646 \\
20-34 years & $78(74.2)$ & $90(85.7)$ & 0.056 \\
$>$ 35 years & $18(17.1)$ & $3(2.8)$ & 0.001 \\
Education level (years) & $9.8 \pm 0.2$ & $10.6 \pm 0.2$ & 0.017 \\
Marital status (married) & $99(94.2)$ & $89(84.7)$ & 0.043 \\
Family support & $100(95.2)$ & $101(96.1)$ & 1.000 \\
Working women & $52(49.5)$ & $54(51.4)$ & 0.890 \\
Nulliparity & $33(31.4)$ & $43(40.9)$ & 0.196 \\
\hline
\end{tabular}

Some clinical significant variables were: number of previous pregnancies, being higher in women with stillbirths, $\mathrm{p}=0.033$; history of stillbirth, $\mathrm{p}=0.012$; the number of antenatal visits was higher in the control group, $\mathrm{p}=0.001$; number of death children was $1.35 \pm 0.07$ in the group of cases against $0.17 \pm 0.04$ in the control group, $\mathrm{p}=$ 0.001; medical complications were observed more in those who had fetal death, $p=0.001$; the frequency of women with psycho-emotional disorders presenting stillbirth were $34.2 \%$, compared with controls who obtained $19.0 \%, \mathrm{p}=$ 0.019 ; contraceptive acceptance was less frequent in women who had stillbirth (66.6 vs. $91.4 \%, \mathrm{p}=0.001)$; and women with stillbirth had higher average of psycho-emotional disorders $2.35 \pm 0.3$ vs. $1.33 \pm 0.1, \mathrm{p}=$ 0.004 , (table 2).

Table 2. Clinical variable analysis of both groups. [Mean $\pm S E ; n(\%)]$

\begin{tabular}{llll}
\hline Variable & $\begin{array}{l}\text { Cases } \\
\mathbf{n = 1 0 5}\end{array}$ & $\begin{array}{l}\text { Controls } \\
\mathbf{n = 1 0 5}\end{array}$ & $\mathbf{p}$ \\
\hline Previous pregnancies & $2.4 \pm 0.1$ & $2.05 \pm 0.1$ & 0.033 \\
Total previous losses & $25(24.8)$ & $15(14.3)$ & 0.082 \\
Previous abortions & $17(16.1)$ & $15(14.3)$ & 0.114 \\
Previous stillbirths & $8(7.6)$ & $0(0)$ & 0.012 \\
Number of antenatal visits & $6.9 \pm 0.3$ & $10.5 \pm 0.4$ & 0.001 \\
Gestation weeks & $30.1 \pm 0.6$ & $38.8 \pm 0.1$ & 0.001 \\
Number death children & $1.35 \pm 0.07$ & $0.17 \pm 0.04$ & 0.001 \\
Medical complications & $35(33.3)$ & $9(8.5)$ & 0.001 \\
Addiction & $5(4.7)$ & $4(3.8)$ & 1.000 \\
Interfamilial violence & $6(5.7)$ & $10(9.5)$ & 0.435 \\
Medical advice & $89(84.7)$ & $91(86.6)$ & 0.844 \\
Contraceptives acceptance & $70(66.6)$ & $96(91.4)$ & 0.001 \\
Psycho-emotional disorders & $36(34.2)$ & $20(19.0)$ & 0.019 \\
\hline
\end{tabular}

In the logistic regression analysis, the only variable who remained statistically significant for the appearance of psycho-emotional disorders was the occurrence of stillbirth, $\mathrm{p}=0.002$. The whole model had a determination coefficient $(\mathrm{R}=0.1071)$, with degree freedom $=14, \mathrm{p}=$ 0.043, (table 3).
Table 3. Logistic regression analysis to determine which sociodemographic and clinical variables are associated with psycho-emotional disorder $(n=210)$.

\begin{tabular}{lllll}
\hline Variable & $\begin{array}{l}\text { Regression } \\
\text { coefficient }\end{array}$ & $\begin{array}{l}\text { Standard } \\
\text { error }\end{array}$ & $\begin{array}{l}\text { Chi square } \\
\text { beta= }\end{array}$ & p \\
\hline Intercept & -14.52 & 2001.09 & 0.00 & 0.994 \\
Age & -2.523 & 3.02052 & 0.70 & 0.403 \\
Education level & -8.442 & 7.20143 & 1.37 & 0.241 \\
Marital status & 0.1787 & 0.60908 & 0.09 & 0.769 \\
Previous abortion & 0.3906 & 0.50670 & 0.59 & 0.440 \\
Previous stillbirth & -0.244 & 0.96927 & 0.06 & 0.800 \\
Number children dead & 0.1666 & 0.75941 & 0.05 & 0.826 \\
Prenatal care & 14.063 & 2001.09 & 0.00 & 0.994 \\
Number of antenatal & 0.0555 & 4.69399 & 1.40 & 0.236 \\
visits & & & & \\
Medical complications & -0.685 & 0.44107 & 2.41 & 0.120 \\
Pregnancy planned & -0.391 & 0.39695 & 0.97 & 0.323 \\
Interfamilial violence & 1.1304 & 0.65633 & 2.97 & 0.085 \\
Family support & -0.598 & 0.86812 & 0.48 & 0.490 \\
Medical advice & 0.4213 & 0.52520 & 0.64 & 0.422 \\
Current stillbirth & 1.3164 & 0.43778 & 9.04 & 0.002 \\
\hline
\end{tabular}

Determination coefficient $(\mathrm{R})$ of the model $=0.1071$, Degree freedom $=14$, Chi square $=23.41, \mathrm{P}=0.043$

In the comparison of the frequency of the psycho-emotional disorders between women with stillbirths and controls, the significant psycho-emotional disorders were: depression, $\mathrm{p}=0.026$; hysteria, $\mathrm{p}=0.012$; psychotic deviation, $p=0.007$; paranoia, $p=0.013$; psychasthenia, $p$ $=0.023$; schizophrenia, $\mathrm{p}=0.017$; low self-esteem, $\mathrm{p}=$ 0.044 ; and anxiety, $\mathrm{p}=0.049$, (table 4 ).

Table 4. Comparison of the psycho-emotional disorders between women with stillbirth and controls, $n(\%)$.

\begin{tabular}{lccc}
\hline $\begin{array}{l}\text { Psycho-emotional } \\
\text { disorder }\end{array}$ & $\begin{array}{c}\text { Cases } \\
\mathbf{n}=\mathbf{1 0 5}\end{array}$ & $\begin{array}{c}\text { Controls } \\
\mathbf{n}=\mathbf{1 0 5}\end{array}$ & $\mathbf{p}$ \\
\hline Hypochondriasis & $9(8.5)$ & $4(3.8)$ & 0.252 \\
Depression & $20(19.0)$ & $8(7.6)$ & 0.026 \\
Hysteria & $8(7.6)$ & $0(0)$ & 0.012 \\
Psychotic deviation & $13(12.3)$ & $2(1.9)$ & 0.007 \\
Feminity & $5(4.7)$ & $8(7.6)$ & 0.567 \\
Paranoia & $20(19.0)$ & $7(6.6)$ & 0.013 \\
Psychasthenia & $23(21.9)$ & $10(9.5)$ & 0.023 \\
Schizophrenia & $25(23.8)$ & $11(10.4)$ & 0.017 \\
Hypomania & $19(18.1)$ & $27(25.7)$ & 0.243 \\
Social introversión & $21(20.0)$ & $11(10.4)$ & 0.084 \\
Anger & $19(18.1)$ & $17(16.1)$ & 0.855 \\
Low self-esteem & $24(22.8)$ & $12(11.4)$ & 0.044 \\
Anxiety & $25(23.8)$ & $13(12.3)$ & 0.049 \\
Posttraumatic stress & $20(19.0)$ & $10(9.5)$ & 0.076 \\
\hline
\end{tabular}

\section{Discussion}

Pregnancy produces not only a series of physiological changes in women, but also includes psychological and social changes. Even when women are cheerful and satisfied with their pregnancy, some women experienced psychological morbidity, especially depression and anxiety in the postpartum period $[15,16]$. Even some studies suggest that psychiatric disorders occurred in $14.1 \%$ of pregnant women. 
It is recognized that fetal loss is perhaps the most difficult and heartbreaking tragedy ever imagined by a woman and her surroundings. Despite being so common, almost nobody talks about this; even at the moment of the confirmation of the diagnosis, the patient perceives a long silence among physicians, who are concentrated and focused on what they observed on the ultrasound screen; the prevalence of this silence during ultrasound examination may, in certain cases, cause further psychological trauma for the mother of a stillborn baby [17]. Despite the society tends to deny the impact, the psychological effects can be devastating [18]. As it has been reported in other studies, our data support that psycho-emotional disorders occur more frequently in patients who have lost their pregnancy by death.

Also our findings agree with other authors [19, 20], who reported that maternal age over 35 years remains as an independent risk factor for stillbirth, even taking into account the medical conditions that are more likely to occur in older women; so it is recommended encourage women get pregnant in early stages of life. Other risk factors associated with fetal death in our study were the stable union, poor prenatal care, the number of previous children dead, low grade of education, and complications of pregnancy, all of them according to other publications [21, 22].

Moreover, it has been reported that the history of miscarriages or previous stillbirths significantly predicted symptoms of depression and anxiety in a subsequent pregnancy [23]. In our study, univariate analysis results were similar, and the antecedent of having previous abortion was a significant variable; other important antecedent was the history of a previous stillbirth, this finding also has been reported by different authors [24, 25], they referred that women who have had stillbirth have poor obstetric prognosis and increases the risk of fetal loss in subsequent pregnancies.

In other study that included 1,339 women, it was found that the presence of posttraumatic stress disorder was associated with higher neuroticism, lower educational level and longer duration of gestation [26]; but our results differ because our women had the resolution on their pregnancies earlier in women with stillbirths. This fact could be explained because the decease of the babies occurred before term. In a prospective study [27], performed to assess the possible psychological disturbances that occurred in women with perinatal loss, the authors found that women with this complication had higher levels of anxiety and depression at immediate postpartum, compared with those women who had a living child. These all findings are similar to our results, because, we found that the fact of experiencing fetal death is a significant predictor for the occurrence of psycho-emotional disorders. But interestingly, we found other psychological disorders in women with stillbirths such as: depression, hysteria, deviation psychotic paranoia, psychasthenia, schizophrenia, low self-esteem and anxiety. This is a new finding that has not been published elsewhere previously, and most publications refer association of anxiety and depression in women with stillbirth, even in the next pregnancy. So we recommend a more exhaustive psychological evaluation in women who have stillbirth in order to offer a more comprehensive support.

Other interesting finding in our women was that of the fourteen variables both socio-demographic and clinical included in the logistic regression analysis, only the presence of stillbirth was significant associated to psycho-emotional disorders; this fact, remarks the strong association between stillbirth as predictive variable and psycho-emotional disorder as outcome variable; so it is needed an active and early intervention giving psychological support to women with stillbirth in order to relief their suffering.

Our study has showed the vulnerability of women with fetal death, to experience negative psychological consequences, which leads to develop adequate psychological care with the intervention of a multidisciplinary team trained and psychological support programs to help solve the duel [28]. Even it has been reported that not only women, but both parents are affected and profoundly altered, so it is recommended psychological support to the couple [29].

In a previous investigation performed by our group in the same setting, we found that women who suffered spontaneous abortion had less frequency of psycho-emotional disorders [30] than women who had stillbirth in our present investigation; this is very interesting because according to the present results, the frequency of psycho-emotional disorders found is more than twice fold in women who had death of their babies with pregnancies in advances stages of gestation. This fact could be explained, in part, due to women who had more weeks of gestation had more favorable expectative about the of outcome of the pregnancy, inclusive many women buy accessories for the future newborn, also the parents talk about the name of the near newborn; and even some couples near to term of the pregnancy discuss about the professional career of the future newborn. But tragically when the baby dies, all that favorable expectative become a devastating experience. Therefore there are more types of psycho-emotional disorders that are found in those women. So could be needed a longer and more comprehensive psychological support in women who had loss of their newborns nearly at term of pregnancy.

\section{Conclusion}

There is higher frequency of psycho-emotional disorders in women, who suffer fetal death, so it is recommended to provide care and early psychological counseling in those women and in their partners. 


\section{References}

[1] Panduro J, Pérez J, Panduro E, Castro J, Vázquez M. Factores de riesgo prenatales en la muerte fetal tardía, Hospital Civil de Guadalajara, Mexico. Rev Chil Obstet Ginecol 2011; 76: 169-174

[2] Cousens S, Blencowe H, Stanton C, Chou D, Ahmed S, Steinhardt L, Creanga A, Tuncalp O, Patel Z, Gupta S, Say L, Lawn J. National, regional, and worldwide estimates of stillbirth rates in 2009 with trends since 1995: a systematic analysis. Lancet 2011; 377: 1319-1330

[3] Lawn J, Blencowe H, Pattinson R, Cousens S, Kumar R, Ibiebele I, Gardosi J, Day L, Stanton C. Stillbirths: Where? when? why? How to make the data count? Lancet 2011; 377: $1448-1463$

[4] American College of Obstetricians and Gynecologists. Management of stillbirth. ACOG Practice Bulletin No. 102 Obstet Gynecol 2009; 113: 748-761

[5] Gardosi J, Madurasinghe V, Williams M, Malik A, Francis A. Maternal and fetal risk factors for stillbirth: population based study. BMJ 2013; 346: 108

[6] Warshak C, Wolfe K, Russell K, Habli M, Lewis D, De Franco E. Influence of adolescence and obesity on the rate of stillbirth. Paediatric and Perinatal Epidemiology 2013; 27: 346-352

[7] Flenady V, Koopmans L, Middleton P, Froen JF, Smith G, Gibbons K, Coory M, Gordon A, Ellwood D, McIntyre H, Fretts R, Ezzati M. Major risk factors for stillbirth in high-income countries: a systematic review and meta-analysis. Lancet 2011; 377: 1331-1340

[8] Radestad I, Steineck G, Nordin C, Sjogren B. Psychological complications after stillbirth-influence of memories and immediate management: population based study. BMJ 1996; 312: $1505-1508$

[9] Kersting A, Wagner B. Complicated grief after perinatal loss. Dialogues Clin Neurosci 2012; 14: 187-194

[10] Blackmore E, Coté D, Tang W, Glover V, Evans J, Golding J, O'Connor T. Previous prenatal loss as predictor of perinatal depression and anxiety. Br J Psychiatry 2011; 198: 373-378

[11] Engerlhard I, Van Den Hout M, Arntz A. Posttraumatic stress disorder after pregnancy loss. Gen Hosp Psychiatry 2001; 23: 62-66

[12] Butcher JN, Dahlstrom WG, Graham JR, Tellegen A, Kaemmer B. The Minnesota Multiphasic Personality Inventory-2 (MMPI-2): Manual for administration and scoring. Minneapolis, MN: University of Minnesota Press, 1989.

[13] D’Orazio L, Meverowitz B, Korst L, Romero R, Goodwin T. Evidence against a link between hyperemesis gravidarum and personality characteristics from an ethnically diverse sample of pregnant women: a pilot study. J Womens Health (Larchmt) 2011; 20: 137-144

[14] Hintze J. Number Cruncher Statistical System. Kaysville, Utah: Statistical Software, 2005. Accessed December 6, 2012.Available at http://www.ncss.com.
[15] Arranz L, Gaviño S, Escobedo F. Depresión posparto en perdida gestacional recurrente. Presentación de un caso. Rev Esp Med 2005; 10: 71-73

[16] Urbina C, Villaseñor S. Los trastornos mentales y el embarazo. Rev Dig Univ 2005; 6: 1-6

[17] Radestad I, Malm MC, Lindgren H, Pettersson K, Larsson LL. Being alone in silence - Mothers' experiences upon confirmation of their baby's death in utero. Midwifery 2014; 30: e91-95

[18] Vargas J, Pacheco A. Óbito fetal, distimia y reacción depresiva. Centro Regional de Investigación en Psicología 2011; 5: 49-55

[19] MacDorman M, Kirmeyer S,Wilson E. Fetal and perinatal mortality, United States, 2006. Natl Vital Stat Rep 2012; 60: $1-23$

[20] Fretts R. Etiology and prevention of stillbirth. Am J Obstet Gynecol 2005; 193: 1923-1935

[21] Reddy U. Prediction and prevention of recurrent stillbirth. Obstet Gynecol 2007; 110: 1151-1164

[22] Gravensteen I, Helgadottir L, Jacobsen E, Sandset P, Ekeberg Ø. Long-term impact of intrauterine fetal death on quality of life and depression: a case-control study. BMC Pregnancy Childbirth 2012; 7: 43

[23] Blackmore E, Coté-Arsenault D, Tang W, Glover V, Evans J, Golding J, O'Connor T. Previous prenatal loss as predictor of perinatal depression and anxiety. $\mathrm{Br} \mathrm{J}$ Psychiatry 2011; 198: 373-378

[24] Frias A, Luikenaar R, Sullivan A, Lee R, Porter T, Branch D, Silver R. Poor obstetric outcome in subsequent pregnancies in women with prior fetal death. Obstet Gynecol 2004; 104: $521-526$

[25] Rivas E, Vasquez D. Óbito fetal: Hallazgos de patología en una institución de alta complejidad. Cartagena, Colombia, 2010-2011. Rev Col Obs y Gin 2012; 63: 376-381

[26] Engerlhard I, Van Den Hout M, Schouten E. Neuroticism and low educational level predict the risk of posttraumatic stress disorder in women after miscarriage or stillbirth. Gen Hosp Psychiatry 2006; 28: 414-417

[27] Carrera L. Papel del obstetra ante la pérdida perinatal. Tesis Doctoral. Universidad de Valencia, Facultad de Medicina 1995: 1-281.

[28] Gausia K, Moran A, Ali M, Ryder D, Fisher C, Koblinsky M. Psychological and social consequences among mothers suffering from perinatal loss: perspective from a low income country. BMC Public Health 2011; 9: 451

[29] Mills T, Ricklesford C, Cooke A, Heazell A, Whitworth M, Lavender T. Parents' experiences expectations of care in pregnancy after stillbirth or neonatal death: a metasynthesis. BJOG 2014; Mar 4, (Epub ahead of print)

[30] Romero-Gutiérrez G, Pérez-Durán N, Camarillo-Trujillo A, Ruiz-Treviño A. Psycho-emotional disorders in women after spontaneous abortion. Clinical Medicine Research 2013; 2: 6-10. 